\title{
Review on Estimation of Road Quality using Mobile Sensors \& Machine Learning Techniques
}

\author{
Trupti K Dange ${ }^{1 *}$, K.H.Walse ${ }^{2}$ and V.M.Thakare ${ }^{3}$ \\ ${ }^{1}$ Assistant Professor,Department of Computer Engineering, \\ RMD Sinhgad School of Engineering,Pune,M.S,India \\ ${ }^{2}$ Professor \& Head, Department of Computer Engineering, \\ Anuradha Engineering College, Chikhli,Buldhana, M.S, India \\ ${ }^{3}$ Professor \& Head, P.G Department of Computer Science,Sant \\ Gadge Baba Amravati University, Amravati, M.S.,India
}

\section{ABSTRACT}

Machine learning techniques have become an integral part of realizing smart transportation Machine learning learns the latent patterns of historical data to model the behaviour of a system and to respond accordingly in order to automate the analytical model building. Using AI based Machine Learning Algorithms can detect the road anomalies, analyse them, share this information to the users while driving and also repair them by sending the relevant information to the road maintenance authorities. The availability of increased computational power and collection of the massive amount of data have redefined the value of the machine learning-based approaches for addressing the emerging demands and needs in transportation systems. The overall objective of this work is to develop an efficient machine learning techniques for mobile sensor data. As road maintenance is costly and authorities cannot go on each and every road network, such applications will be useful to provide the data to the higher authorities so that they can take the valuable actions towards it.Machine learning for sensors and signal data is becoming easier than ever: hardware is becoming smaller and sensors are getting cheaper. The paper presents brief information about the smart phone sensors and techniques used in Machine Learning how they are used for the road anomaly detection and the work done up till now in that domain

KEY WORDS: ROAD MONITORING, ACCELEROMETER, GYROSCOPE, MACHINE LEARNING, ANDROID

\section{INTRODUCTION}

Road network is the key transport infrastructure for any country. They are paramount for country's economic growth and development and social benefits. According to statistics US has the world's largest road network size followed by India on 2nd rank and China on 3rd

\section{ARTICLE INFORMATION}

*Corresponding Author: trupti.dange@gmail.com

Received 11th Oct 2020 Accepted after revision $27 n d$ Dec 2020

Print ISSN: 0974-6455 Online ISSN: 2321-4007 CODEN: BBRCBA

Thomson Reuters ISI Web of Science Clarivate Analytics USA and Crossref Indexed Journal

\section{1) Clarivate $\begin{gathered}\text { Analytics } \\ \text { rossef }\end{gathered}$}

NAAS Journal Score 2020 (4.31)

A Society of Science and Nature Publication,

Bhopal India 2020. All rights reserved.

Online Contents Available at: http//www.bbrc.in/

Doi: $h$ ttp://dx.doi.org/10.21786/bbrc/13.14/55 rank. But as the road network size is increasing in the developing countries than the developed countries road safety is also crucial. According to the WHO (World Health Organization) of 2020 approximately 1.35 million people die each year due to road crashes with highest death rate in the African region. Poor road infrastructure, distracted driving, pothole, uneven roads, etc. are also responsible for the death rates. These problems cannot get resolved by just adding a greater number of roads as the vehicle production and sales are increasing day-by-day.Above addressed road safety measures can get improved using the new technologies. As this is the digital era, technology makes crucial contribution to the society. Past few decades have given so many solutions to the transportation domain to increase its efficiency and effectiveness. 
Technologies that provided tremendous roles areMachine Learning (ML) Algorithm and IoT (Internet of Things) that provided various ways to overcome the problems of the transportation domain. The data that is collected for these technologies are mostly the Mobile sensors data collected continuously for analysis or the sensors designed to be used externally with the vehicles.

2. Data Sensing using Smartphone Sensors: In today's world, technology has made a drastic change to the society that has changed the standard of living of humans. Smartphone is one of the biggest examples of technology. Each and every human now days are able to get the smartphones at affordable rates. Using smartphones, we are able to collect a lot of data either related to person's lifestyle, surroundings or location. These smartphones play an important role for collecting the data related to the transportation that does help to resolve the issues faced by the transportation systems. This data is collected using the in-built sensors of the smartphones. As discussed in the previous sections, the road safety conditions problems can get resolved using the smartphone sensors. We can detect the potholes, bumps, uneven roads, etc. using these sensors.

To detect this problem, we have to continuously acquire the data from the smartphone sensors so that enough data can be processed to obtain the better results. Sensors that are used to acquire the data are accelerometer, gyroscope, magnetometer, GPS etc.To use these sensors the vehicle directions and smartphone directions must be same. That is, the smartphone should be mounted properly while driving the vehicle. If the smartphone is kept stable inside the vehicle it may lead to incorrect data collection. In AzzaAlloch,2017[Ravi Bhoraskar ,2012] [Aniket Kulkarni,2014][Gaurav Singal ,2018][Yun-chin Tai 1 ,2010][Arun Kumar ,2017] the smartphone and vehicle axes are made fixed so that the correct data should get obtained.

2.1Accelerometer: Accelerometer is an electromechanical device used for measuring acceleration forces. This sensor is used to sense the movement or vibration. It is the measurement of change of velocity or speed divided by time. In [AzzaAlloch,2017][Ravi Bhoraskar ,2012] [Aniket Kulkarni,2014][Gaurav Singal ,2018][Yun-chin Tai 1 ,2010][Arun Kumar ,2017] this sensor of smartphone (3-axes) is used to get the change in the driving speed of the vehicle. Using this sensor data, we can find out that whether the vehicle is driving on the smooth road surface or any bump, pothole or speed breaker has occurred on the road surface. The smartphone accelerometers are dynamic accelerometer which measures gravitational force also. This can be removed from the collected data by using many techniques.

2.2Gyroscope: Gyroscope is the sensor which is used to maintain a reference direction or provide a stability in navigation. The gyroscope in smartphone is used to check the orientation of the smartphone inside the vehicle. The orientation of smartphone can change the accelerometer data which may lead to wrong prediction for the road condition [AzzaAlloch,2017][Aniket Kulkarni,2014]. If the smartphone in the vehicle is not mounted properly there are many mathematical calculations that can be used to change the unstable smartphone directions data into stable direction data.which has been shown in Table 1

2.3GPS: GPS is a radio navigation system. It is used to find the longitude and latitude of a device if the device has a GPS tracker active on the smartphone. The GPS system plays an important role while detecting the location of the unwanted activity while driving a vehicle. While driving if it is detected that the accelerometer and gyroscope captured some unwanted activity while driving, then using GPS system of the smartphone [AzzaAlloch,2017][Ravi Bhoraskar ,2012] [Aniket Kulkarni,2014][Gaurav Singal ,2018][Yun-chin Tai 1,2010$]$ [Arun Kumar ,2017 we can see the location of the unwanted activity like pothole, bump, slope, speed breaker, etc. on the road surface.

\subsection{Challenges of smartphone sensors:}

2.4.1. Vibration pattern of sensor data: The sensor readings depend on the speed of the vehicle that is, how it approached and what was the orientation of the sensor therefore given pothole or any road anomaly not necessarily give the same pattern during each drive. If the vehicles suspension system is not the normal condition then large vibration may experience by the vehicle which may lead to more deviation.[Lei Kang,2017 ] [Arun Kumar ,2017]

2.4.2 Benign event: There are events like door slams, railroad crossing, any object under the tires must not be considered as the potholes or any other uneven road condition discussed above. [Lei Kang,2017 ][Arun Kumar ,2017

2.4.3 GPS error: GPS system has an error of 3 meters which may lead to incorrect identification of the longitude and latitude to detect the anomaly of the road. [Lei Kang,2017 ][Arun Kumar ,2017

2.4.4 Privacy: As we are going to use the GPS system to detect the location of the pothole or any anomaly on the road, we need the access to the user's smartphone which may lead to privacy breach of the user.[Lei Kang,2017 ] [Arun Kumar ,2017].

3.Estimation technique to detect road quality: Machine Learning is a part of Artificial Intelligence (AI) and is a data-driven solution that can cope with the new system requirements. IoT is a system of interrelated computing devices that transfers data over the network without human-to-human or human-to-computer interaction. Many authors used these technologies to analyse the road conditions in transportation.

3.1 XSense: Lei Kang and Suman Banerjee, proposed Practical Driving Analytics with Smartphone Sensors. This paper basically focuses on the several techniques which can be used to improve the accuracy and usability 
of inertial sensors. The author discussed various problems and error that can arise due to the inertial sensors that may mislead the correct identification of the pothole on the road surfaces. The XSense application is designed by the author on the android platform. The application uses accelerometer, gyroscope and GPS sensors of the smartphone to collect the data. The author used novel techniques that can increase the accuracy of these sensors in more extent; the techniques like detecting orientation change, conducting slope-aware, coordinate alignment and linear acceleration estimation. Moving variance method and Intra-cluster variance method are used to detect the orientation change and estimate the mounting stability of the smartphone. The author also addressed the GPS data when used combined which machine learning techniques give high accuracy for detection. [Lei Kang,2017]

\subsection{Road Quality Management System using Mobile} Sensors: Arun Kumar G et. al. proposed a Road Quality Management System using Mobile Sensors. The author used the accelerometer and GPS sensors to identify the pothole and bumps and detect its location accordingly. The author has created such a system with four parts; sensing, storing, analysing and retrieving. Cloud computing concept is used by the author to store the information regarding the potholes to get easily accessible to the higher authorities. To identify the pothole, a threshold value is set, if the value changes then it is identified as the pothole or bump on the road surface. Data analysing is done using a web application in this system. The cloud is made public accessible so that smartphone users get the pothole located on their mobile phones. [Arun Kumar ,2017].

3.3 RoadSense: AzzaAlloch et. al designed a RoadSense Android application to estimate the road condition using accelerometer, gyroscope and GPS sensors. Potholle detection methodsThe proposed system is divided into two parts; training and prediction phases. . The data is labelled into two categories; soothed and potholed. Once the data is collected using the accelerometer and gyroscope sensors, they are passed to the training phase. In training phase, a low pass filter is applied on the collected data to remove the high frequencies and new accelerometer and gyroscope magnitude values are obtained. The feature extraction is done where a vector of Fourier coefficients is obtained. The author used the Correlation-based Feature Selection approach to select the features from the previously obtained vector. The author uses C4.5 Decision Tree Algorithm, SVM and Naïve Bayes for prediction from which the C4.5 Decision Tree algorithm gives the highest accuracy of $98.50 \%$ for correctly predicting the potholes. [AzzaAlloch,2017]

Table 1. Smartphone Sensors to detect Road Quality

\begin{tabular}{|c|c|c|c|c|c|}
\hline \multirow[t]{2}{*}{$\begin{array}{l}\text { Sr. } \\
\text { NO }\end{array}$} & \multirow{2}{*}{ System } & \multicolumn{4}{|c|}{ Smartphone Sensors } \\
\hline & & $\mathbf{A}$ & $\mathbf{G}$ & $\mathbf{M}$ & GPS \\
\hline 1 & RoadSenseAzzaAlloch, 2017 & $\checkmark$ & $\checkmark$ & & $\checkmark$ \\
\hline 2 & Wolveriene[Ravi Bhoraskar,2012] & 2 & & 2 & \\
\hline 3 & Encog[Aniket Kulkarni,2014] & 2 & & & \\
\hline 4 & $\begin{array}{l}\text { Pothole [6][A1i } \quad \text { Tizghadam } \\
\text { Detection using Machine Learning }\end{array}$ & $\checkmark$ & $\checkmark$ & & $\checkmark$ \\
\hline 5 & PitFree[Gaurav Singal ,2018] & 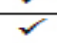 & 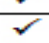 & & $\checkmark$ \\
\hline 6 & Xsense [Yun-chin Tai 1,2010$]$ & $\checkmark$ & & & $\checkmark$ \\
\hline 7 & MI using AI Mohammad Abbadi & 2 & $\checkmark$ & & $\checkmark$ \\
\hline 8 & Streetcheck [15] & $\checkmark$ & & & $\checkmark$ \\
\hline 9 & $\begin{array}{ll}\text { MII } & \text { using } \\
\text { IOT Sensors[JIn Ren Ng] }\end{array}$ & & & & \\
\hline
\end{tabular}

3.4 Pitfree: Gaurav Singal et. Al. propose a model Pitfree which detects the pothole on road using mobile sensors. The author has collected the data using accelerometer (3 - axes) to get the data regarding road condition and GPS system data to identify the longitude and latitude of the pothole on the road surface. The collected data is clustered using K-means clustering algorithm into two categories (smoothed and pothole). The author analyses the accelerometer for normal driving condition, pothole condition and speed breaker condition. According to author, when the graph first goes down and then goes up signifies that detection of the pothole. Total of 250 samples were used for classification. Three classifiers are used to identify the pothole, SVM, Random Forest and Logistic Regression from which the SVM classifier gives the highest frequency of $99.6 \%$ for correctly identifying the potholes. [Gaurav Singal ,2018].

Ali Tizghada et. al. enlightens about how machine learning approaches are being used in the transportation domain and how massive amount of data have redefined these approaches for emerging demands and needs in transportation. The paper briefly addresses the transportation issues that can be solved using machine learning approaches such as prediction of future traffic conditions, public transit commuter's travel 
pattern, detect vehicle and track vehicle trajectories in traffic conditions, identity of the public transport driver, challenges in air travel like collision avoidance, automated decision support technologies, etc. The author also focuses on the aircraft transport issues and how Synthetic Minority Oversampling Technique (SMOTE) and Ensemble-Based Ordinal Classification approach can resolve these issues. [Ali Tizghadam ,2019].

3.5 Road Condition using IOT Sensors: Jin Ren Ng et. Al. proposed a research work for road surface condition identification using IoT sensors and Machine Learning approach. The author instead of relying on the smartphone sensors used IoT sensors accelerometer (3-axes) with Arduino microcontroller placed to the front wheels of the four-wheeler vehicle. The author collected total of 350 sets with seven different road surface conditions which includes smooth road, speed bump, pothole, rumble strip, uneven road, sudden.

stops and turns. The feature extraction and selection are done for both time and domain frequency with some statistical parameters. The author used Ranker, Greedy Algorithm and Particle Swarm Optimization (PSO) techniques for feature selection and K-Nearest Neighbour, Random Forest and SVM for classification. The performance of the classifiers is measured using Correctly Classified Rate (CCR). The system gave 99\% of the accuracy for all the classifiers expect SVM (Polynomial) which gives the accuracy of 94\%. [JIn Ren Ng].

Table 2. Assesment of Machine Learning Technique to estimate Road quality

\begin{tabular}{|c|c|c|c|c|c|c|c|c|}
\hline \multirow[t]{2}{*}{ Sr.No } & \multirow[t]{2}{*}{ System } & \multicolumn{6}{|c|}{ Machine Learning Model } & \multirow[t]{2}{*}{ Accuracy } \\
\hline & & DT & KNN & $\begin{array}{l}\mathbf{N} \\
\mathbf{N}\end{array}$ & RF & LR & NB & \\
\hline 1 & $\begin{array}{l}\text { RoadSenseAzz } \\
\text { aAlloch,2017 }\end{array}$ & 2 & & & & & 2 & $98.60 \%$ \\
\hline 2 & $\begin{array}{l}\text { Wolveriene[Ra } \\
\text { vi Bhoraskar }\end{array}$ & & 2 & & & & & - \\
\hline 3 & Encog[Aniket & & & $\checkmark$ & & & & $95 \%$ \\
\hline 4 & $\begin{array}{l}\text { Pothole [6][A1i } \\
\text { Tizghadam } \\
\text { 2019][ A.S. } \\
\text { E1-Wakeel] }\end{array}$ & 2 & & & & & 2 & $\begin{array}{c}\text { More } \\
\text { than } 80 \%\end{array}$ \\
\hline 5 & $\begin{array}{l}\text { PitFree[Gaura } \\
\text { v Singal }\end{array}$ & & & 2 & 2 & 2 & 2 & $99.60 \%$ \\
\hline 6 & $\begin{array}{l}\text { Xsense [Yun- } \\
\text { chin Tai } 1\end{array}$ & 2 & & 2 & 2 & 2 & 2 & $90 \%$ \\
\hline 7 & $\begin{array}{l}\text { MI using AI } \\
\text { Mohammad } \\
\text { Ahbadi }\end{array}$ & 2 & & 2 & 2 & 2 & 2 & $99.90 \%$ \\
\hline 8 & $\begin{array}{l}\text { Streetcheck } \\
\text { MNunesand Mot }\end{array}$ & & & $\checkmark$ & 2 & & & $97 \%$ \\
\hline 9 & MII using & & $\checkmark$ & & 2 & & & $99 \%$ \\
\hline
\end{tabular}

The various methods in Machine Learning with combined AI approach can also be used for the analysis as shown in Mohammad Abbadi to provide more accuracy to system.. Even though the presented work gives you correct prediction for road conditions but there are many ways to try to solve this problem to a bigger extent which will be useful for the higher authorities for road maintenance.

\section{CONCLUSION \&t FUTURE WORK}

This study highlights the use of smartphone sensors and machine learning algorithm using AI technique. Many authors have used various algorithms for the prediction of data. Each algorithm plays an important role in every presented work by different authors. Some authors also used some mathematical techniques to optimize the sensors data so that correct data must be captured and there should be no misleading outputs. The paper provides a good description of the various methods and its performance. fusing AIMohammad
Abbadi procedures with Smartphone sensors .Using some techniques of Machine Learning (Neural network, KNN, Naïve Bayes and Random forest tree) to prepare and test information received from Smartphone sensors. All inspected AI calculations keep up high precision and recall in obtaining the vehicle's driving class; be that as it may, the neural network classifiers accomplish the most noteworthy precision value which is $90 \%$.

Governments' representative architects and laborers are unable to alert about harm to streets, potholes, and so on. This is profoundly tedious and requires a great number of labourers. Consequently, it would fill in as a notice framework to all clients just as an information base of potholes with their areas to the experts for activity. Study analysis the exhibition of different AI models (Logistic Regression, Support Vector Machine (SVM)Mohammad Abbadi, K-Nearest Neighbours (KNN), Naive Bayes, Decision Tree, Random Forest and Ensemble Voting) in view of various output parameters (Accuracy, F-score, Precision and Recall) and distinguished that 
Random Forest is the best model for pothole recognition Et combination of two or more sensors provide better accuracy and scalability to system.

\section{REFERENCES}

AzzaAlloch et. al., "RoadSense: Smartphone Application to Estimate Road Condition using Acceleromter and Gyroscope”, IEEE Sensors Journal, Vol.17 Issue 13, July 1, 2017 ,pp: 4231-4238

Arun Kumar et. al., "Road Quality Management System using Mobile Sensors”, International Conference on Innovations in Information Embedded and Communication Systems, 2017.

Ali Tizghadam et. al., "Machine Learning in Transportation”, Hindawi, Journal of Advanced Transportation, Vol. 2019.

A. S. El-Wakeel, A. Osman, A. Noureldin, and H. S. Hassanein, "Road test experiments and statistical analysis for real-time monitoring of road surface conditions," in -2017 IEEE Global Communications Conference. IEEE, 2017, pp. 1-6.

Aniket Kulkarni et. al., "Pothole Detection System using Machine Learning on Android”, International Journal of Emerging Technology and Advance Engineering, Vol. 4 Issue 7, July 7, 2014, pp: 360-364,.

FotiosZantalis et. al., "A Review of Machine Learning and IoT in Smart Transportation”, Future Internet, 2019.

Gaurav Singal et. al., "Pitfree: Pot-holes detection on Indian Roads using Mobile Sensors", IEEE 8th International Advance Computing Conference, 2018. H. Sharma, S. Naik, A. Jain, R. K. Raman, R. K. Reddy, and R. B. Shet,"S-road assist: Road surface conditions and driving behavior analysis using smartphones," in 2015 International Conference on Connected Vehicles and Expo (ICCVE). IEEE, 2015, pp. 291-296.
JIn Ren Ng et. al., "Identification of Road Surface Condition using IoT Sensors and Machine Learning”, Computational Science and Technology, Lecture Notes in Electrical Engineering, Vol. 481

Jinting Ren et.al,’PADS: A Reliable Pothole Detection System"Using Machine Learning” Springer International Publishing AG 2017pp. 327-338, 2017. DOI: 10.1007/9783-319-52015-5 33

Lei Kang et.al.,"Practical Driving Analytics with Smartphone Sensors" IEEE Vehicular Networking Conference (VNC),2017.

Lei Kang and Suman Banerjee, "Practical Driving Analytics with Smartphone Sensors”, IEEE Vehicular Networking Conference, 2017, pp: 303-310.

Mohammad Abbadi, et.al.," Machine Learning-Based Approach For Detecting Driver Behavior Using Smartphone Sensors ",INTERNATIONAL JOURNAL OF SCIENTIFIC \& TECHNOLOGY RESEARCH VOLUME 8, ISSUE 12, DECEMBER 2019

NunesandMota et.al.," A participatory sensing framework to classify road surface quality Journal of Internet Services and Applications “,(2019) 10:13 https://doi.org/10.1186/s13174-019-011119

P. Mohan, V. N. Padmanabhan, and R. Ramjee, "Nericell: rich monitoring of road and traffic conditions using mobile smartphones," in Proceedings of the 6th ACM conference on Embedded network sensorsystems. ACM, 2008, pp. 323-336.

Ravi Bhoraskar et. al., "Wolverine: Traffic and road condition estimation using smartphone sensors", Fourth International Conference on Communication Systems and Networks, 2012.

Yun-chin Tai et. al., "Automatic road anomaly detection using smart mobile device", 15th Conference on Artifical Intelligence and Applications, 2010 